\title{
OPEN Novel plasma biomarkers improve discrimination of metabolic health independent of weight
}

\begin{abstract}
Stephen Ellison ${ }^{1,4}$, Jawan W. Abdulrahim ${ }^{2,4}$, Lydia Coulter Kwee ${ }^{2}$, Nathan A. Bihlmeyer ${ }^{2}$, Neha Pagidipati ${ }^{3}$, Robert McGarrah ${ }^{2,3}$, James R. Bain ${ }^{2}$, William E. Kraus ${ }^{2,3}$ \& Svati H. Shah ${ }^{2,3 凶}$

We sought to determine if novel plasma biomarkers improve traditionally defined metabolic health $(\mathrm{MH})$ in predicting risk of cardiovascular disease (CVD) events irrespective of weight. Poor $\mathrm{MH}$ was defined in CATHGEN biorepository participants ( $n>9300)$, a follow-up cohort ( $>5600$ days) comprising participants undergoing evaluation for possible ischemic heart disease. Lipoprotein subparticles, lipoprotein-insulin resistance (LP-IR), and GlycA were measured using NMR spectroscopy $(n=8385)$, while acylcarnitines and amino acids were measured using flow-injection, tandem mass spectrometry $(n=3592)$. Multivariable Cox proportional hazards models determined association of poor $\mathrm{MH}$ and plasma biomarkers with time-to-all-cause mortality or incident myocardial infarction. Low-density lipoprotein particle size and high-density lipoprotein, small and medium particle size (HMSP), GlycA, LP-IR, short-chain dicarboxylacylcarnitines (SCDA), and branched-chain amino acid plasma biomarkers were independently associated with CVD events after adjustment for traditionally defined MH in the overall cohort $\left(p=3.3 \times 10^{-4}-3.6 \times 10^{-123}\right)$, as well as within most of the individual BMI categories $\left(p=8.1 \times 10^{-3}-1.4 \times 10^{-49}\right)$. LP-IR, GlycA, HMSP, and SCDA improved metrics of model fit analyses beyond that of traditionally defined MH. We found that LP-IR, GlycA, HMSP, and SCDA improve traditionally defined $\mathrm{MH}$ models in prediction of adverse CVD events irrespective of BMI.
\end{abstract}

Claiming more lives in 2015 than the combined mortality of the second and third leading causes, cancer and chronic lower respiratory disease, respectively, cardiovascular disease (CVD) remains the leading cause of morbidity and mortality within the United States ${ }^{1}$. Obesity, a known risk factor for a variety of negative health effects including CVD and adverse CVD-related events ${ }^{2}$, remains a major public health challenge; in fact, the age-adjusted percentage of adults greater than 20 years old with obesity in the US increased from $22.9 \%$ in 1988-1994 to $37.8 \%$ in $2013-2014^{3}$. The relationship between obesity and CVD has been partially attributed to the increased prevalence of intermediate risk factors including insulin resistance, type 2 diabetes mellitus (DM), dyslipidemia, and hypertension. However, the underpinnings remain incompletely elucidated and the heterogeneity of prevalence of these risk factors in obesity demonstrates the complexity of the relationship ${ }^{4}$.

Metabolic health $(\mathrm{MH})$ has traditionally been defined by the presence or absence of a cluster of intermediate CVD risk factors known as metabolic syndrome ${ }^{5}$, while medical literature has also classically defined being overweight and having poor metabolic health as synonymous ${ }^{6}$. Metabolic syndrome is a known contributor to the epidemic of $\mathrm{CVD}^{7}$, however, a growing body of evidence suggests that weight is a poor metric of metabolic health ${ }^{4,8}$ even though obesity continues to be reported as a "major determinant of metabolic syndrome".

While intermediate CVD risk factors seen in metabolic syndrome are important, perhaps the most important metric for defining metabolic health should be its ability to associate with hard cardiovascular endpoints. Poor metabolic health as defined by metabolic syndrome is associated with increased risk of major adverse cardiovascular events (MACE) in the overall population ${ }^{7}$, but evidence suggests the risk of MACE is not well correlated with BMI category ${ }^{10}$, often as a result of underestimating the risk of those patients who have components of metabolic syndrome but do not meet full criteria ${ }^{11,12}$.

In order to more effectively target interventions to improve metabolic health and prevent adverse CVD events, there is a need for better markers of metabolic health beyond traditionally defined $\mathrm{MH}$ that are predictive regardless of BMI. Plasma biomarkers reflect dysregulated systemic and tissue-specific metabolism, serving as downstream read-outs of genetic, transcriptomic and proteomic variation, and thus may serve as these better

${ }^{1}$ Department of Anesthesiology, Duke University Medical Center, Durham, NC, USA. ${ }^{2}$ Duke Molecular Physiology Institute, Duke University School of Medicine, 300 North Duke St, Durham, NC 27701, USA. ${ }^{3}$ Division of Cardiology, Department of Medicine, Duke University School of Medicine, Durham, NC, USA. ${ }^{4}$ These authors contributed equally: Stephen Ellison and Jawan W. Abdulrahim. ${ }^{\circledR}$ email: svati.shah@duke.edu 
markers. In fact, recent studies in our lab using metabolomic profiling have identified a number of biomarkers that are associated with obesity, insulin resistance, DM, CVD, and response to interventions, including low-density lipoprotein (LDL-P) $)^{13-15}$, lipoprotein-insulin resistance (LP-IR) ${ }^{16}$, GlycA ${ }^{17}$, sum of medium plus small high-density lipoprotein-particle subclass (HMSP) ${ }^{18}$, short-chain dicarboxylacylcarnitines (SCDA) ${ }^{19,20}$, and branched-chain amino acids (BCAA) ${ }^{20-22}$. Thus, we hypothesized that these novel plasma biomarkers can improve predictive capabilities for incident adverse CVD events across BMI categories and are better markers of metabolic health than traditional measures which often rely heavily on BMI.

\section{Methods}

CATHGEN biorepository participants $(\mathrm{n}>9300)$, a follow-up cohort (>5600 days) comprising participants undergoing evaluation for possible ischemic heart disease.

Study population. Individuals undergoing cardiac catheterization between 2001 and 2010 for the indication of possible ischemic heart disease at Duke University Medical Center provided written consent and enrolled in the CATHGEN (CATHeterization GENetics) biorepository $(n>9300)$ as previously described ${ }^{23}$. For this follow-up cohort, longitudinal annual follow-up for recurrent events, hospitalizations, and vital status has been performed and confirmed through the National Death Index and the Social Security Death Index for $>5600$ days from time of CATHGEN enrollment. CATHGEN is approved by the Duke University School of Medicine Institutional Review Board in accordance with the Declaration of Helsinki (Durham, NC, USA). Prior to administration of supplemental heparin, $50 \mathrm{~mL}$ of blood was collected at the time of arterial puncture from fasting, consented participants. Samples were immediately cooled to $4{ }^{\circ} \mathrm{C}$, processed to separate plasma, and then frozen to $-80^{\circ} \mathrm{C}$. Clinical data including demographics, medical comorbidities, angiographic data, quantitation of left ventricular ejection fraction (LVEF), and laboratory data were collected. For this study, participants were excluded if they were cardiac transplant recipients $(n=201)$, had undergone cardiac catheterization for the indication of pulmonary hypertension $(n=94)$, had a history of congenital heart disease $(n=27)$, had missing BMI $(n=1)$, had no longitudinal follow-up data $(n=8)$, had missing biomarker data $(n=115)$, or had incomplete data necessary for calculation of traditionally defined $\mathrm{MH}(\mathrm{n}=226)$.

Race was classified as white or non-white. LVEF was obtained by ventriculogram at the time of cardiac catheterization or, if ventriculography was not performed, by an echocardiogram or cardiac magnetic resonance image (MRI). Coronary artery disease was determined by the physician performing the cardiac catheterization, and defined as present if a stenosis $\geq 50 \%$ was present in any major epicardial coronary vessel. For this study, the primary endpoint was defined as time-to-event for all-cause mortality or MI from CATHGEN enrollment.

Definition of BMI categories and traditionally defined poor $\mathrm{MH}$. Individuals were classified into three categories based on BMI at time of enrollment: lean $\left(\mathrm{BMI}<25 \mathrm{~kg} / \mathrm{m}^{2}\right)$, overweight (BMI $\left.25-30 \mathrm{~kg} / \mathrm{m}^{2}\right)$, or obese $\left(\mathrm{BMI} \geq 30 \mathrm{~kg} / \mathrm{m}^{2}\right)$. Individuals were categorized as having traditionally defined poor $\mathrm{MH}$ if they had at least two out of four criteria for poor $\mathrm{MH}$ at baseline enrollment, and considered to have traditionally defined good $\mathrm{MH}$ if they met $0-1$ criteria (modified slightly from the Adult Treatment Panel III (ATPIII) guidelines ${ }^{24}$ given lack of waist circumference data in CATHGEN): (1) fasting triglycerides (TG) $\geq 150 \mathrm{mg} / \mathrm{dL}$, (2) HDL $<40 \mathrm{mg} /$ $\mathrm{dL}$ in men or $<50 \mathrm{mg} / \mathrm{dL}$ in women, (3) history of diabetes, insulin use, fasting glucose $\geq 100 \mathrm{mg} / \mathrm{dL}$, or HOMAIR $>5.13$, (4) blood pressure medication use or history of patient reported hypertension. Homeostatic model assessment of insulin resistance was calculated as follows: HOMA-IR $=\left(\right.$ fasting insulin in $(\mu \mathrm{UI} / \mathrm{mL}){ }^{*}$ glucose $(\mathrm{mM}) / 22.5^{21,25}$. A HOMA-IR threshold of $>5.13$ (i.e., the 90 th percentile) was selected and consistent with prior work $^{21}$.

Metabolomic profiling. Lipoprotein particle concentrations, sizes, and subclasses as well as GlycA, LP-IR, and creatinine were measured as previously described ${ }^{17,20,26}$. For this study, we used a composite sum of medium plus small HDL-particle subclass (diameters $\leq 9.4 \mathrm{~nm}$, termed HMSP) because we have previously reported HMSP as more strongly associated with incident CVD events than individual HDL components ${ }^{17}$. GlycA is an inflammatory biomarker that consists of the NMR signal from the $N$-acetyl methyl groups of the $N$-acetylglucosamine residues on enzymatically glycosylated acute phase proteins (primarily al-antichymyotrypsin, $\alpha 1$-acid glycoprotein, haptoglobin, al-antitrypsin, and transferrin $)^{17}$. The LP-IR score is a measure of insulin resistance derived using the weighted sum of six NMR derived lipoprotein parameters ${ }^{16}$; the multiplex validated score ranges from 0 to 100 (where 100 is the most insulin resistant).

For measurement of acylcarnitines and amino acids, targeted metabolic profiling was performed using flowinjection, tandem mass spectrometry (MS/MS) as previously reported ${ }^{27}$. Values below the lower limits of quantitation (LLOQ) were reported and analyzed as "0," while plasma biomarkers with $>25 \%$ of values below the LLOQ were not analyzed.

Principal components analysis (PCA) with varimax rotation was used for reduction of metabolic data into identifiable factors, with those factors having an eigenvalue of $\geq 1.0$ being retained, and plasma biomarkers with a factor load of $\geq 0.4$ being reported as comprising a factor as previously described ${ }^{20,27,28}$. In our prior work, a PCA-derived factor composed of SCDA (glutaryl carnitine (C5-DC), 3-hydroxy-cis-5-octenoyl carnitine or hexenedioyl (C6:1-DC/C8:1-OH), octenedioyl carnitine (C8:1-DC), adipoyl carnitine (C6-DC), methylmalonyl carnitine or succinyl carnitine (Ci4-DC/C4-DC), 3-hydroxy-decanoyl carnitine or suberoyl carnitine (C10-OH/ C8-DC), 3-hydroxy-dodecanoyl carnitine or sebacoyl carnitine (C12-OH/C10-DC), and citrulline) is predictive of incident CVD events. Thus, that PCA factor was used in this study. Prior work has also shown a PCA-derived factor of BCAA and two aromatic amino acids is associated with CAD and adverse CVD events; thus, that PCA factor was also used in this study (composed of leucine/isoleucine, valine, phenylalanine, tyrosine, and 
methionine $)^{27}$. These PCA-factors for SCDA and BCAA are used as measures for the composite of SCDA and BCAA in this study.

Statistical methods. Baseline clinical and demographic characteristics were compared between individuals with and without traditionally defined poor $\mathrm{MH}$ using $t$-tests and chi-squared tests for continuous and categorical variables, respectively. Incident events were defined as all-cause mortality or MI (adjudicated by a central process within the Duke Databank for Cardiovascular Disease). Cox proportional hazards models were used to examine the independent and additive effects of plasma biomarkers on time-to-event, accounting for traditionally defined poor MH. Specifically, we tested three models for time-to-event, each adjusted for BMI category (lean, overweight, obese) in the full sample: (1) traditionally defined poor $\mathrm{MH}$; (2) each individual plasma biomarker alone (LDL-P, LP-IR, GlycA, HMSP, BCAA, and SCDA), adjusted for traditionally defined poor $\mathrm{MH}$; and (3) a full model inclusive of traditionally defined poor $\mathrm{MH}$ and all plasma biomarkers jointly. We also stratified the data by BMI category, and tested the same models to understand the potential heterogeneity of effects of the relationship of traditionally defined poor $\mathrm{MH}$ and novel plasma biomarkers across BMI categories. To evaluate medications as a confounder, we performed sensitivity analyses on a subset of individuals with available medication data, where time-to-event models were adjusted for statin use. Statin use was determined using EHR admission medications, i.e. an individual was considered to be on a statin if they were on a statin at admission either 365 days prior to or 10 days after the index cardiac catheterization. Finally, while our primary goal was to identify better markers of metabolic health compared to traditionally defined MH parameters (using association with incident events as the metric to determine "better"), in sensitivity analyses, we also created models that included traditional CVD risk factors (age, sex, race, smoking, ejection fraction, LDL-C, creatinine, family history of $\mathrm{CAD}$, and presence/absence of CAD). The assumption of proportional hazards was tested for each model; when violated, we included a term in the model with a time-varying coefficient and compared the resulting inference to inference based on the original model. We used the Akaike Information Criterion (AIC) and area under the receiver operating characteristic curve (AUC) to compare model fit between different models and determine the incremental discriminative capability of novel plasma markers. AIC analyses were restricted to individuals who had data needed for all models of traditionally defined MH and for all biomarkers $(n=3222)$.

\section{Results}

Table 1 shows baseline characteristics of the overall study population ( $\mathrm{n}=8671$ individuals) stratified by both metabolic health and BMI categories; 1921 (22.2\%) individuals were classified as lean, 3068 individuals (35.4\%) were overweight, and 3682 individuals (42.5\%) were obese. Obese individuals tended to be younger than overweight or lean individuals (59.2 \pm 11.2 years vs. $62.8 \pm 11.7$ years vs. $64.1 \pm 13.1$ years, respectively) and had greater prevalence of races other than white. Approximately $45 \%$ of obese and lean individuals were female compared with only $30 \%$ of overweight individuals.

Overall, there was a high prevalence of traditionally defined poor $\mathrm{MH}$ (71.3\%). As expected, obese and overweight individuals had a greater prevalence of poor $\mathrm{MH}$ compared with lean individuals (81.5\% vs. $69.1 \%$ vs. $55.3 \%$ ), although the prevalence of poor $\mathrm{MH}$ was high even in the lean group. Concurrently, $18.5 \%$ of obese individuals were defined as having good $\mathrm{MH}$ by traditional parameters, further confirming the known disconnect between metabolic health and weight. The increasing prevalence of poor $\mathrm{MH}$ across increasing BMI categories was driven by all components of the metabolic health measure including higher prevalence of hypertension and diabetes, higher TG, and lower HDL. Interestingly, LDL was lower in poor MH individuals as compared with good MH individuals across each of the three BMI categories ( $96.0 \mathrm{vs} .102 .9 \mathrm{mg} / \mathrm{dL}$ lean, $101.3 \mathrm{vs} .106 .7 \mathrm{mg} / \mathrm{dL}$ overweight, 101.4 vs. $111.9 \mathrm{mg} / \mathrm{dL}$ obese, poor vs good $\mathrm{MH}$ individuals respectively).

In the overall cohort, individuals with poor metabolic health had a higher prevalence of a positive family history of CAD as compared with individuals with good metabolic health $(34.4 \%$ vs. $29.9 \%, p<0.001)$, which was also true in the overweight category $(35.2 \%$ vs. $31.3 \%, p<0.05)$. However, there were no significant differences in the lean and obese group.

Association of BMI and traditionally defined poor $\mathrm{MH}$ with incident CVD events. There were 3845 death or MI events (44.3\% of individuals; $6.5 \%$ MI, $37.8 \%$ deaths) with a mean time to event of 2433 days (SD 1263 days). Traditionally defined poor MH was associated with time-to-death/MI in the overall cohort (HR $1.38,95 \%$ CI $1.28-1.49, \mathrm{p}=5.0 \times 10^{-17}$, Fig. $1 \mathrm{~A}$ ). In the same models stratified by BMI category, traditionally defined poor $\mathrm{MH}$ was associated with a stepwise increase in HR for increasing BMI category: lean: HR 1.23, 95\% CI 1.08-1.39, $p=1.4 \times 10^{-3}$; overweight: HR 1.45, 95\% CI 1.28-1.64, $p=7.2 \times 10^{-9}$; obese: HR 1.53, 95\% CI $1.32-1.77, p=2.0 \times 10^{-8} .1028$ events occurred in the lean population (54\% of lean individuals), with 411 events occurring in lean individuals with traditionally defined good $\mathrm{MH}$.

Interestingly, after accounting for smoking history, obesity was protective for events, with higher BMI categories associated with a lower risk of events (overweight: HR: 0.71, 95\% CI 0.65-0.77; obese: HR 0.65, 95\% CI 0.59-0.70, both compared to referent lean BMI category) (Fig. 1B).

Novel plasma biomarkers predict incident events independent of traditionally defined poor $\mathrm{MH}$ and across all BMI categories. To understand the prognostic capability of novel plasma biomarkers independent of traditional metabolic health parameters, we analyzed Cox models for each plasma biomarker with time-to-death/MI, adjusting for traditionally defined poor $\mathrm{MH}$ (Table 2). In these analyses, each plasma biomarker was significantly associated with time-to-death/MI in the overall cohort; GlycA and SCDA were associated with decreased survival, while LDL-P, LP-IR, HMSP and BCAA were all associated with increased survival. In analyses stratified by BMI category, each individual plasma biomarker remained significantly pre- 


\begin{tabular}{|c|c|c|c|c|c|c|c|c|}
\hline & \multicolumn{8}{|c|}{ Stratified by BMI category } \\
\hline & \multicolumn{2}{|l|}{ Overall } & \multicolumn{2}{|c|}{ Lean $(\mathrm{BMI}<25)$} & \multicolumn{2}{|c|}{ Overweight (BMI 25-30) } & \multicolumn{2}{|c|}{ Obese $(\mathrm{BMI} \geq 30)$} \\
\hline & Good MH & Poor MH & Good MH & Poor MH & Good MH & Poor MH & Good MH & Poor MH \\
\hline $\mathrm{n}(\%)$ & $2486(28.7)$ & $6185(71.3)$ & $858(44.7)$ & $1063(55.3)$ & $947(30.9)$ & $2121(69.1)$ & $681(18.5)$ & $3001(81.5)$ \\
\hline Age (years) & $61.4(12.5)$ & $61.6(11.7)$ & $62.5(13.5)$ & $65.4(12.7)^{\star *}$ & $62.0(11.8)$ & $63.1(11.6)^{*}$ & $59.1(12.0)$ & $59.3(11.0)$ \\
\hline Female (\%) & 40.7 & $37.3^{*}$ & 47.3 & $43.1^{* *}$ & 30.8 & 29.7 & $46.3^{*}$ & $40.8^{*}$ \\
\hline BMI $\left(\mathrm{kg} / \mathrm{m}^{2}\right)$ & $27.7(6.2)$ & $31.1(7.4)^{* *}$ & $22.2(2.1)$ & $22.6(2.0)^{\star *}$ & $27.3(1.4)$ & $27.6(1.4)^{\star \star *}$ & $35.2(6.1)$ & $36.7(6.6)^{* *}$ \\
\hline White (\%) & 78.8 & $73.2^{* *}$ & 80.3 & 78.1 & 81.2 & $77.8^{*}$ & 73.6 & $68.2^{\star}$ \\
\hline Hypertension (\%) & 42.4 & $90.3^{* *}$ & 39.0 & $90.2^{* *}$ & 43.7 & $88.3^{* *}$ & 44.6 & $91.8^{\star *}$ \\
\hline Diabetes (\%) & 8.6 & $67.3^{* *}$ & 8.0 & $57.9^{* \star}$ & 9.2 & $62.8^{* *}$ & 8.5 & $73.7^{* *}$ \\
\hline Glucose (mg/dL) & $97.4(21.8)$ & $126.6(53.7)^{* *}$ & $95.7(23.3)$ & $117.6(49.2)^{* *}$ & $98.0(20.2)$ & $122.7(50.0)^{* *}$ & $98.7(21.7)$ & $132.5(57.0)^{* *}$ \\
\hline HOMA-IR & $1.22(1.30)$ & $2.42(2.65)^{* *}$ & $0.84(0.99)$ & $1.50(2.00)^{\star *}$ & $1.23(1.34)$ & $2.05(2.25)^{\star *}$ & $1.70(1.45)$ & $3.03(2.97)^{*}$ \\
\hline Smoking (\%) & $1112(44.7)$ & $2970(48.0)^{*}$ & $412(48.0)$ & $530(49.9)$ & $422(44.6)$ & $1059(49.9)^{*}$ & $278(40.8)$ & $1381(46.0)^{*}$ \\
\hline LVEF\% & $57.0(12.5)$ & $54.4(14.3)^{* *}$ & $56.3(13.0)$ & $52.7(15.9)^{* *}$ & $57.4(12.3)$ & $54.2(14.2)^{\star *}$ & $57.4(12.3)$ & $55.2(13.8)^{* *}$ \\
\hline CAD (\%) & $1229(52.8)$ & $4188(70.2)^{* *}$ & $384(48.9)$ & $740(73.3)^{* *}$ & $529(58.6)$ & $1541(74.8)^{* *}$ & $316(49.5)$ & $1907(65.9)^{* *}$ \\
\hline $\begin{array}{l}\text { Family history of CAD } \\
(\%)\end{array}$ & 744 (29.9) & $2128(34.4)^{* *}$ & $247(28.8)$ & $343(32.3)$ & $284(30.0)$ & $730(34.4)^{*}$ & $213(31.3)$ & $1055(35.2)$ \\
\hline $\mathrm{HDL}(\mathrm{mg} / \mathrm{dL})$ & $54.4(18.0)$ & $41.6(13.0)^{* *}$ & $59.5(21.4)$ & $44.3(15.0)^{* *}$ & $52.3(15.7)$ & $41.5(12.5)^{\star *}$ & $50.9(14.4)$ & $40.8(12.4)^{* *}$ \\
\hline LDL (mg/dL) & $106.7(36.5)$ & $100.4(40.9)^{* *}$ & $102.9(34.9)$ & $96.0(39.8)^{*}$ & $106.7(37.1)$ & $101.3(40.6)^{* \star}$ & $111.9(37.2)$ & $101.4(41.3)^{* \star}$ \\
\hline Creatinine (mg/dL) & $1.08(1.03)$ & $1.27(1.34)^{* *}$ & $1.11(1.38)$ & $1.36(1.66)^{* *}$ & $1.08(0.80)$ & $1.30(1.37)^{\star *}$ & $1.06(0.77)$ & $1.22(1.18)^{* \star}$ \\
\hline $\mathrm{TG}(\mathrm{mg} / \mathrm{dL})$ & $127.1(92.5)$ & $189.2(193.8)^{* *}$ & $106.9(68.5)$ & $151.1(110.0)^{* *}$ & $133.9(92.9)$ & $176.4(141.8)^{* *}$ & $144.1(113.0)$ & $211.0(239.5)^{* \star}$ \\
\hline \multicolumn{9}{|l|}{ Follow-up } \\
\hline MI (\%) & $110(4.4)$ & $453(7.3)^{* *}$ & $36(4.2)$ & $59(5.6)$ & $46(4.9)$ & $177(8.3)^{\star *}$ & $28(4.1)$ & $217(7.2)^{*}$ \\
\hline Death (\%) & $883(35.5)$ & $2710(43.8)^{* *}$ & $398(46.4)$ & $594(55.9)^{* *}$ & $300(31.7)$ & $935(44.1)^{* *}$ & $185(27.2)$ & $1181(39.4)^{* *}$ \\
\hline Time to death/MI (days) & $2526(1278)$ & $2450(1307)^{* *}$ & 2305 (1287) & $2231(1341)^{* *}$ & 2539 (1228) & $2438(1268)^{* *}$ & $2630(1185)$ & $2458(1239)^{\star \star}$ \\
\hline
\end{tabular}

Table 1. Baseline characteristics of the CATHGEN study population stratified by traditionally defined $\mathrm{MH}$ and BMI category. ${ }^{*} p<0.05,{ }^{* *} p<0.001$ for comparison to good $\mathrm{MH}$. All continuous variables reported as mean (SD). $M H$ Traditionally defined metabolic health, BMI body mass index, HOMA-IR homeostatic model assessment-insulin resistance, $L V E F$ left ventricular ejection fraction, $C A D$ coronary artery disease, $H D L$ highdensity lipoprotein, $L D L$ low-density lipoprotein, $T G$ triglycerides, $M I$ myocardial infarction.

dictive of death/MI and with similar effect sizes across each BMI category except BCAA, which was associated with time-to-event only in obese individuals. Models for plasma biomarkers LDL-P, LP-IR, GlycA, and HMSP included 8385 individuals, and those for BCAA and SCDA included 3591 individuals.

Sensitivity analyses including individuals with medication data $(\mathrm{N}=3,174$ out of 8761 [36.2\%]) were conducted including statin use as a binary covariable (Supplementary Table 1). Overall, the magnitude and significance of results remained the same in these analyses.

In a full Cox model inclusive of traditionally defined poor $\mathrm{MH}$ and all plasma biomarkers, each individual plasma biomarker remained independently significant with a similar magnitude of effect in the overall cohort (Supplementary Table 2). In similar full models stratified by BMI category, each plasma biomarker other than LP-IR and LDL-P also remained significantly predictive of events in one or more obesity categories, with GlycA, HMSP, and SCDA maintaining significance across all BMI categories (Supplementary Table 2).

The proportional hazards assumption was violated in our models for plasma biomarkers LP-IR, GlycA, and HMSP in the overall population and the BMI-stratified analyses. To examine the potential impact of non-proportional hazards related to these plasma biomarkers, we conducted sensitivity analyses allowing for a time-varying coefficient in the model. All plasma biomarkers were still significantly associated with events in all tested models $\left(p<10^{-12}\right)$. Estimates of the HR at various timepoints are given in Supplementary Table 3; these demonstrate that the magnitude of the estimated HR for each plasma biomarker increases when the time-varying coefficient is included in the model, compared to the results in Table 2. Therefore, for simplicity in the remaining analyses and with the understanding that this may lead to an underestimation of the effect sizes for LP-IR, GlycA, and HMSP, we utilized a Cox model without time-varying coefficients.

Novel plasma biomarkers independently predict events in full multivariable models additionally adjusted for clinical risk factors. While our primary goal was to understand novel plasma biomarkers as markers of metabolic health using event prediction as a benchmark for assessing importance, there are also other known clinical risk factors for incident CVD events that are not captured by models of traditionally defined poor $\mathrm{MH}$; thus, we also created full multivariable models, including age, sex, race, LVEF, smoking, LDL$\mathrm{C}$, creatinine, and presence/absence of CAD. In these models, which also adjusted for traditionally defined poor $\mathrm{MH}$, each individual novel plasma biomarker except BCAA remained predictive of events in the overall population (Table 2). In BMI category stratified analyses, LP-IR, GlycA, and HMSP plasma biomarkers were significantly and consistently associated with incident CVD events across all BMI categories with a similar magnitude 

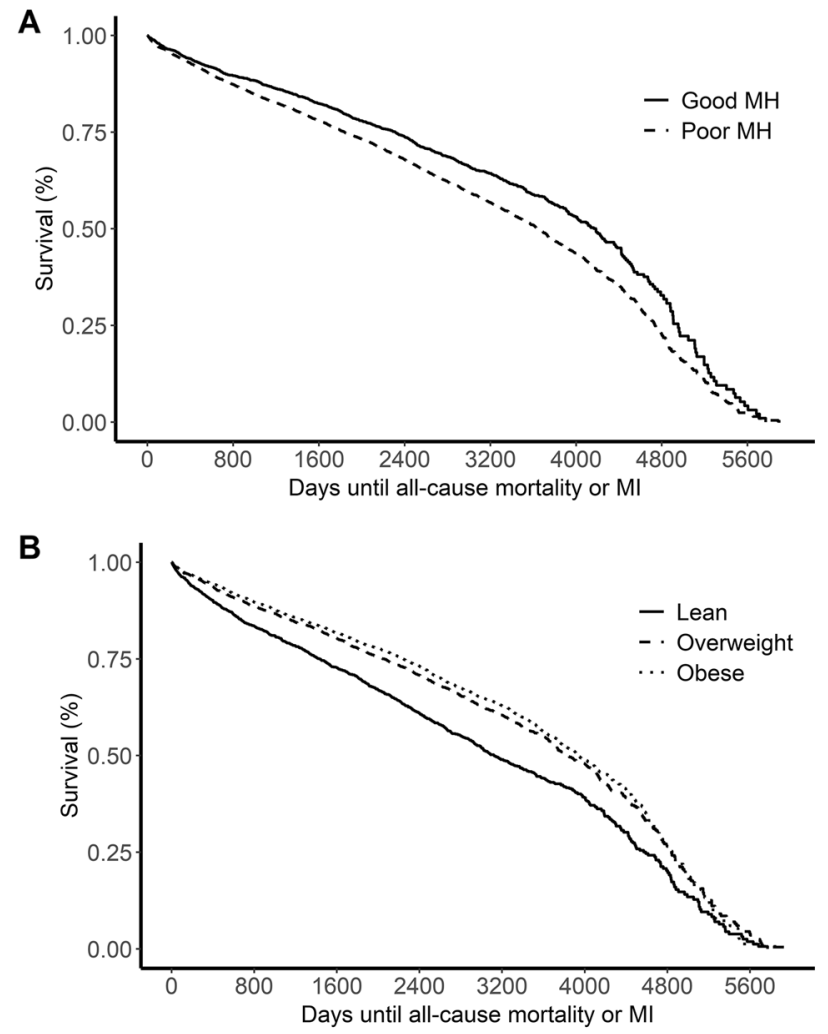

Figure 1. Unadjusted Kaplan-Meier plots for relationship between time-to-death or incident MI and metabolic health (A) and BMI category (B).

\begin{tabular}{|c|c|c|c|c|c|c|c|c|}
\hline \multirow[b]{2}{*}{ Model $^{*}$} & \multicolumn{2}{|l|}{ Overall cohort } & \multicolumn{2}{|l|}{ Lean } & \multicolumn{2}{|l|}{ Overweight } & \multicolumn{2}{|l|}{ Obese } \\
\hline & HR (95\% CI) & $p$ value & HR (95\% CI) & $p$ value & HR (95\% CI) & $p$ value & HR (95\% CI) & $p$ value \\
\hline \multicolumn{9}{|l|}{ Unadjusted model } \\
\hline MH & $1.38(1.28-1.49)$ & $4.5 \times 10^{-17}$ & $1.23(1.08-1.39)$ & $1.4 \times 10^{-3}$ & $1.45(1.28-1.64)$ & $7.2 \times 10^{-9}$ & $1.53(1.32-1.77)$ & $2.0 \times 10^{-8}$ \\
\hline $\mathrm{MH}+\mathrm{LDL}-\mathrm{P}$ & $0.91(0.88-0.94)$ & $2.5 \times 10^{-8}$ & $0.91(0.85-0.97)$ & $5.3 \times 10^{-3}$ & $0.88(0.83-0.94)$ & $3.3 \times 10^{-5}$ & $0.93(0.88-0.98)$ & $8.1 \times 10^{-3}$ \\
\hline $\mathrm{MH}+\mathrm{LP}-\mathrm{IR}$ & $0.78(0.75-0.81)$ & $3.1 \times 10^{-40}$ & $0.75(0.70-0.82)$ & $3.3 \times 10^{-12}$ & $0.79(0.74-0.84)$ & $2.5 \times 10^{-14}$ & $0.79(0.75-0.83)$ & $1.3 \times 10^{-17}$ \\
\hline $\mathrm{MH}+$ GlycA & $1.27(1.24-1.31)$ & $2.9 \times 10^{-59}$ & $1.28(1.21-1.35)$ & $6.2 \times 10^{-19}$ & $1.32(1.26-1.39)$ & $2.8 \times 10^{-27}$ & $1.23(1.17-1.29)$ & $2.4 \times 10^{-16}$ \\
\hline $\mathrm{MH}+\mathrm{HMSP}$ & $0.66(0.64-0.68)$ & $3.6 \times 10^{-123}$ & $0.67(0.63-0.72)$ & $9.6 \times 10^{-34}$ & $0.63(0.60-0.67)$ & $1.4 \times 10^{-49}$ & $0.67(0.63-0.71)$ & $1.1 \times 10^{-44}$ \\
\hline $\mathrm{MH}+\mathrm{BCAA}$ & $0.91(0.86-0.96)$ & $3.3 \times 10^{-4}$ & $0.94(0.85-1.04)$ & 0.23 & $0.93(0.85-1.02)$ & 0.13 & $0.87(0.80-0.94)$ & $6.3 \times 10^{-4}$ \\
\hline $\mathrm{MH}+\mathrm{SCDAs}$ & $1.25(1.20-1.30)$ & $5.1 \times 10^{-29}$ & $1.15(1.06-1.24)$ & $6.9 \times 10^{-4}$ & $1.3(1.23-1.38)$ & $3.7 \times 10^{-19}$ & $1.31(1.22-1.41)$ & $2.5 \times 10^{-13}$ \\
\hline \multicolumn{9}{|l|}{ Fully adjusted model $^{\star *}$} \\
\hline $\begin{array}{l}\text { MH + clinical covari- } \\
\text { ates }^{* *}\end{array}$ & $1.21(1.11-1.31)$ & $5.3 \times 10^{-6}$ & $1.04(0.9-1.19)$ & 0.61 & $1.22(1.07-1.40)$ & $3.6 \times 10^{-3}$ & $1.53(1.3-1.81)$ & $4.4 \times 10^{-7}$ \\
\hline $\begin{array}{l}\mathrm{MH}+\mathrm{LDL}-\mathrm{P}+\text { clini- } \\
\text { cal covariates }^{* *}\end{array}$ & $0.8(0.73-0.87)$ & $7.2 \times 10^{-7}$ & $0.72(0.6-0.87)$ & $4.6 \times 10^{-4}$ & $0.71(0.61-0.83)$ & $1.5 \times 10^{-5}$ & $0.97(0.84-1.12)$ & 0.66 \\
\hline $\begin{array}{l}\text { MH + LP-IR + clini- } \\
\text { cal covariates** }\end{array}$ & $0.83(0.80-0.87)$ & $1.1 \times 10^{-18}$ & $0.8(0.73-0.87)$ & $8.7 \times 10^{-7}$ & $0.84(0.78-0.90)$ & $4.6 \times 10^{-7}$ & $0.84(0.78-0.89)$ & $3.2 \times 10^{-8}$ \\
\hline $\begin{array}{l}\mathrm{MH}+\text { GlycA + clini- } \\
\text { cal covariates }\end{array}$ & $1.27(1.23-1.31)$ & $1.6 \times 10^{-49}$ & $1.25(1.18-1.33)$ & $9.1 \times 10^{-14}$ & $1.3(1.24-1.38)$ & $1.1 \times 10^{-21}$ & $1.24(1.18-1.31)$ & $2.0 \times 10^{-16}$ \\
\hline $\begin{array}{l}\mathrm{MH}+\mathrm{HMSP}+\text { clini- }^{-} \\
\text {cal covariates }\end{array}$ & $0.71(0.68-0.74)$ & $1.4 \times 10^{-70}$ & $0.68(0.63-0.73)$ & $2.7 \times 10^{-24}$ & $0.71(0.66-0.76)$ & $2.2 \times 10^{-25}$ & $0.72(0.68-0.77)$ & $4.0 \times 10^{-25}$ \\
\hline $\begin{array}{l}\mathrm{MH}+\mathrm{BCAA}+\text { clini- } \\
\text { cal covariates }\end{array}$ & $0.94(0.89-1.00)$ & 0.051 & $0.92(0.81-1.03)$ & 0.14 & $0.97(0.88-1.08)$ & 0.62 & $0.95(0.87-1.04)$ & 0.29 \\
\hline $\begin{array}{l}\mathrm{MH}+\mathrm{SCDAs}+\text { clini- }^{-} \\
\text {cal covariates }\end{array}$ & $1.24(1.14-1.34)$ & $9.2 \times 10^{-7}$ & $1.23(1.06-1.41)$ & $4.7 \times 10^{-3}$ & $1.36(1.19-1.55)$ & $6.2 \times 10^{-6}$ & $1.05(0.87-1.28)$ & 0.61 \\
\hline
\end{tabular}

Table 2. Hazard ratios, confidence intervals, and $p$ values for death or incident MI prediction for traditionally defined poor $\mathrm{MH}$ and novel plasma biomarkers in the overall cohort and stratified by BMI category. ${ }^{*} \mathrm{MH}$ models included 8671 samples; LDL-P, LP-IR, GlycA, and HMSP models included 8385 samples; BCAA and SCDA models included 3591 samples. ${ }^{* *}$ Clinical covariates include: age, sex, race, LVEF, CAD, family history of CAD, smoking, LDL-C, and creatinine. 


\begin{tabular}{|c|c|c|c|c|c|c|c|c|}
\hline \multirow[b]{2}{*}{ Model $^{\mathbf{a}}$} & \multicolumn{2}{|c|}{ Overall } & \multicolumn{2}{|l|}{ Lean } & \multicolumn{2}{|c|}{ Overweight } & \multicolumn{2}{|l|}{ Obese } \\
\hline & $\mathrm{AIC}^{*}$ & AUC & AIC $^{*}$ & AUC & AIC $^{*}$ & AUC & AIC $^{*}$ & AUC \\
\hline \multicolumn{9}{|l|}{ Without clinical covariates } \\
\hline $\mathrm{MH}$ & 20,240 & 0.58 & 4343 & 0.55 & 5846 & 0.56 & 7038 & 0.54 \\
\hline $\mathrm{MH}+\mathrm{LDL}-\mathrm{P}$ & 20,222 & 0.59 & 4343 & 0.55 & 5837 & 0.57 & 7032 & 0.55 \\
\hline $\mathrm{MH}+\mathrm{LP}-\mathrm{IR}$ & 20,179 & 0.61 & 4332 & 0.59 & 5831 & 0.60 & 7006 & 0.59 \\
\hline $\mathrm{MH}+$ GlycA & 20,162 & 0.63 & 4315 & 0.62 & 5821 & 0.63 & 7016 & 0.60 \\
\hline $\mathrm{MH}+\mathrm{HMSP}$ & 20,051 & 0.64 & 4300 & 0.63 & 5782 & 0.65 & 6952 & 0.62 \\
\hline $\mathrm{MH}+\mathrm{BCAA}$ & 20,232 & 0.61 & 4344 & 0.57 & 5846 & 0.61 & 7032 & 0.59 \\
\hline $\mathrm{MH}+\mathrm{SCDA}$ & 20,155 & 0.62 & 4329 & 0.57 & 5807 & 0.62 & 7010 & 0.59 \\
\hline $\mathrm{MH}+$ all plasma biomarkers & 19,919 & 0.69 & 4269 & 0.68 & 5745 & 0.69 & 6907 & 0.67 \\
\hline \multicolumn{9}{|l|}{ With clinical covariates ${ }^{*}$} \\
\hline $\mathrm{MH}+$ clinical covariates & 19,676 & 0.75 & 4241 & 0.71 & 5645 & 0.76 & 6780 & 0.75 \\
\hline MH + LDL-P + clinical covariates & 19,675 & 0.75 & 4243 & 0.72 & 5643 & 0.76 & 6782 & 0.75 \\
\hline $\mathrm{MH}+\mathrm{LP}-\mathrm{IR}+$ clinical covariates & 19,660 & 0.75 & 4239 & 0.72 & 5644 & 0.76 & 6771 & 0.75 \\
\hline $\mathrm{MH}+$ GlycA + clinical covariates & 19,599 & 0.76 & 4210 & 0.74 & 5619 & 0.78 & 6761 & 0.76 \\
\hline $\mathrm{MH}+\mathrm{HMSP}+$ clinical covariates & 19,561 & 0.76 & 4207 & 0.73 & 5613 & 0.77 & 6728 & 0.76 \\
\hline $\mathrm{MH}+\mathrm{BCAA}+$ clinical covariates & 19,674 & 0.75 & 4241 & 0.72 & 5647 & 0.76 & 6781 & 0.75 \\
\hline $\mathrm{MH}+\mathrm{SCDA}+$ clinical covariates & 19,651 & 0.75 & 4236 & 0.72 & 5630 & 0.76 & 6781 & 0.75 \\
\hline $\mathrm{MH}+$ all plasma biomarkers + clinical covariates & 19,482 & 0.77 & 4180 & 0.75 & 5588 & 0.78 & 6711 & 0.77 \\
\hline
\end{tabular}

Table 3. Comparison of model fit characteristics overall and stratified by BMI category. ${ }^{\star}$ AIC analyses were restricted to individuals who had data needed for all models of traditionally defined $\mathrm{MH}$ and for all biomarkers $(n=3222)$. Clinical covariates include: age, sex, race, LVEF, CAD, family history of CAD, smoking, LDL-C, and creatinine.

of effect in each category, suggesting they are important biomarkers regardless of BMI. Traditionally defined poor $\mathrm{MH}$ remained predictive overall and in the overweight and obese categories, but was not significant in its prediction of events in the lean group (Table 2).

In full multivariable models inclusive of traditionally defined poor $\mathrm{MH}, \mathrm{CVD}$ clinical risk factors, and all plasma biomarkers, four plasma biomarkers were significant in the overall cohort: GlycA (HR 1.23, 95\% CI $1.17-1.29, p=3.6 \times 10^{-18}$ ); HMSP (HR 0.76, 95\% CI 0.71-0.81, $p=2.0 \times 10^{-18}$ ); BCAA (HR 0.93, 95\% CI 0.88-0.99, $p=0.01$ ); and SCDA (HR 1.17, 95\% CI $1.08-1.28, p=2.7 \times 10^{-4}$ ). In analyses stratified by BMI category, only GlycA and HMSP remained significantly predictive of events across all BMI categories (Supplementary Table 4).

Novel plasma biomarkers incrementally improve CVD event prediction. To further assess the incremental predictive capabilities of each novel plasma biomarker, we compared models using the AIC and AUC. These models demonstrated modest improvement of model fit with addition of any individual novel plasma biomarker to traditionally defined poor $\mathrm{MH}$ in the overall cohort: traditionally defined poor $\mathrm{MH}$ (AIC 20,240, AUC 0.58), LDL-P (AIC 20,222, AUC 0.59), LP-IR (AIC 20,179, AUC 0.61), GlycA (AIC 20,162, AUC 0.63), HMSP (AIC 20,051, AUC 0.64), BCAA (AIC 20,232, AUC 0.61), SCDA (AIC 20,155, AUC 0.62) (Table 3). BMI stratified categories showed similar improvement of model fit with addition of any individual novel plasma biomarker other than LDL-P (Table 3). When all plasma biomarkers are included, there is greater improvement in model fit than with any single variable alone in the overall cohort (AIC 19,919, AUC 0.69) and within each BMI category (Table 3).

In models inclusive of clinical covariates, we found modest improvement of model performance beyond that of traditionally defined poor $\mathrm{MH}$, with further improvement in performance with addition of any individual plasma biomarker other than LDL-P in the overall cohort: traditionally defined poor MH (AIC 19,674, AUC 0.74), LDL-P (AIC 19,673, AUC 0.74), LP-IR (AIC 19,658, AUC 0.74), GlycA (AIC 19,598, AUC 0.75), HMSP (AIC 19,560, AUC 0.75), BCAA (AIC 19,672, AUC 0.75), SCDA (AIC 19,649, AUC 0.75) (Table 3). BMI stratified categories showed improvement of model fit with addition of GlycA, HMSP, BCAA, and SCDA. Similar to prior, the greatest improvement in model performance occurs in the model inclusive of traditionally defined poor MH, all plasma biomarkers, and clinical covariates (AIC 19,481, AUC 0.77) (Table 3).

We also explored the potential association between the plasma biomarkers and family history of CAD (Supplementary Table 5) and found significant correlations between $\operatorname{LP}-\operatorname{IR}\left(\beta=0.20, p=1.18 \times 10^{-17}\right), \operatorname{LDLP}(\beta=0.07$, $p=0.002)$ and HMSP $\left(\beta=0.12, p=7.49 \times 10^{-7}\right)$ in the overall cohort. In the lean group, only LP-IR $(\beta=0.25$, $\left.p=1.82 \times 10^{-8}\right)$ and HMSP $(\beta=0.16, p=0.002)$ were associated with family history of CAD.

\section{Discussion}

In this large cardiovascular cohort study, we demonstrate that novel plasma biomarkers discovered through prior studies of CVD may serve as better markers of poor metabolic health as determined by their ability to predict risk of adverse cardiovascular events across all BMI categories, independent of, and incremental to, traditionally defined poor $\mathrm{MH}$ parameters. Our most striking results include: (1) traditionally defined poor $\mathrm{MH}$ 
does not predict CVD events in lean individuals in full multivariable models, but the majority of novel plasma biomarkers do; (2) GlycA and HMSP plasma biomarkers had strong predictive capabilities in the overall cohort and across all BMI categories even after adjusting for traditionally defined poor $\mathrm{MH}$ and other clinical risk factors, and with the greatest improvements in AUC on top of traditionally defined poor $\mathrm{MH}$; (3) adding all novel plasma biomarkers to traditionally defined poor MH improved the AUC from 0.58 to 0.69 in the overall cohort, and with a similar magnitude of improvement in lean and overweight BMI categories where metabolic health is perhaps the most heterogeneous.

In our study, $55.3 \%$ of lean individuals met criteria for poor $\mathrm{MH}$, while $18.5 \%$ of obese individuals met criteria for having good $\mathrm{MH}$. Furthermore, of the 3845 total events, $24.3 \%$ occurred in individuals with good $\mathrm{MH}$ and $26.7 \%$ occurred in lean individuals. These highlight the disconnect between traditionally defined poor $\mathrm{MH}$, weight, and risk of morbidity and mortality.

We used hard outcomes of death or incident MI to determine utility of traditional and novel risk factors. Overall, all of the novel plasma biomarkers assessed in this study other than BCAA were found to be predictive of incident CVD events, even after adjusting for traditionally defined poor $\mathrm{MH}$ and additional clinical risk factors. This predictive capability held true in analyses stratified by BMI category for plasma biomarkers LP-IR, GlycA, and HMSP. Furthermore, GlycA and HMSP were strongly predictive of events in the lean group, which is classically felt to be of lesser adverse cardiovascular event risk but may actually have a higher risk. Importantly, novel plasma biomarkers also improved model fit on top of traditionally defined poor MH and additional clinical risk factors, suggesting that they have incremental risk-predictive capabilities beyond what is easily assessed clinically.

Serum concentrations of GlycA, a novel inflammatory biomarker that is an aggregate measure of enzymatically glycosylated, acute-phase proteins, have been shown to independently predict incident CVD events in the MESA study (HR 1.33, 95\% CI 1.24-1.42) and in the JUPITER trial (HR 1.33, 95\% CI $1.21-1.45)^{29,30}$, and with presence and extent of $\mathrm{CAD}^{17}$. The biologic relevance of elevations in GlycA seen in these studies may be due to effects on glycosylation of HDL components resulting in impaired function ${ }^{17}$.

In full multivariate models, a factor composed of SCDA was robust for prediction of death or MI in the overall cohort as well as in lean and overweight individuals. These plasma biomarkers have been shown to be associated with increased risk of adverse CVD related events in the CATHGEN cohort ${ }^{19,20}$, and appear to be reporting on dysregulated endoplasmic reticulum stress ${ }^{28,31}$.

The role of HDL cholesterol in CVD is complex; studies have demonstrated an inverse relationship of HDL-C with CVD, but pharmacologic intervention to increase HDL-C have failed to show improved outcomes ${ }^{32}$. A growing number of studies, however, are showing that NMR-derived lipoprotein particle number more closely correlates with risk of adverse CVD outcomes than traditional LDL-C and HDL-C measures ${ }^{18,33}$. The cardioprotective effects of HMSP seen in the current study likely depend on specific HDL subparticle characteristics that are not well accounted for by traditional HDL-C, which is preferentially affected by larger, cholesterol rich particles ${ }^{32}$; similar to that seen in a recent study showing that the sum of medium- and small-size HDL particles was the strongest predictor of mortality ${ }^{18}$.

In the current study, higher LDL-P levels were protective against death or MI. From prior work with the CATHGEN population, we have seen evidence of a similar lipid paradox ${ }^{13-15,20}$. The etiology of this phenomenon remains incompletely elucidated and may be related to medication use (e.g. patients with higher LDL levels are treated earlier and for longer with statins) but new evidence might suggest that nutritional status plays a key role $^{34}$.

Unexpectedly, while HOMA-IR was higher with increasing BMI, higher LP-IR levels were protective of death or MI. This might be related to the observed obesity paradox in our secondary prevention dataset, i.e. because obesity is protective of adverse outcomes and obese individuals have higher HOMA-IR.

In addition, individuals with poor metabolic health had a higher prevalence of a positive family history of $\mathrm{CAD}$ as compared with individuals with good metabolic health which was also true in the overweight category. However, there were no significant differences in the lean and obese group, suggesting that this more simple screening tool would not be sufficient for defining CVD risk. Moreover, we found correlations between LP-IR, LDLP and HMSP and family history of CAD in the overall cohort. In the lean group, only LP-IR and HMSP were associated with family history of CAD. This is as predicted given the known heritability of these markers.

Regarding the clinical utility of our findings and testing for these plasma biomarkers, it is relatively quick, reproducible, and of course, by their nature, non-invasive ${ }^{35}$. Also, mass spectrometry is currently already in use for new-born screening and more severe metabolic phenotypes (e.g. phenylketonuria, maple-syrup urine disease, etc.). Although the use of some of these plasma biomarkers is still in its infancy, there is a growing body of evidence that supports their clinical utility in cardiovascular disease ${ }^{35}$. Of note, the NMR-derived lipoproteins are commonly used in clinical practice already.

Several strengths of our study deserve mention including the relatively large sample size, availability and length of follow-up data for hard CVD outcomes, fasting samples collected under a rigorous and similar protocol, and the breadth of clinical variables available. Importantly, many prior studies of metabolic health measures do not have a "gold standard" to tag improvement of models to; the use of a clinically relevant outcome of death or $\mathrm{MI}$ is an important metric that we were able to use to compare models. However, since this is a cross-sectional, observational study, we were unable to account for chronic dietary and environmental variations in plasma biomarker levels, nor did we have sufficient medication data to enable adjustment for medication use (although previous work showed such adjustments had minimal influence on plasma biomarker levels $)^{27}$. Finally, although the model of metabolic health improved with the addition of clinical covariates to the plasma biomarkers, the increase was modest, which suggests that clinical covariates contribute more to the models rather than the biomarkers. However, we note that the greatest improvement was seen in the lean group, a group that is often dismissed clinically as having low CVD risk, and as such, biomarkers may have the greatest clinical utility for identifying higher risk individuals in this group. 
In conclusion, with growing evidence of the discordance between metabolic health and BMI, it is crucial to improve clinical models for early recognition and characterization of individuals at high risk for CVD events, and thus at greatest need for early intensive therapeutic intervention. We found plasma biomarkers had the potential to improve clinical models, to better classify overall metabolic health, and to independently predict events, irrespective of obesity stratum. This was especially striking given that traditionally defined $\mathrm{MH}$ models were unable to predict events in the lean group, while novel plasma biomarkers were successful in prediction of events; this suggests that novel plasma biomarkers may be better markers of metabolic health in this patient population than current standard clinical models.

Received: 15 April 2020; Accepted: 18 November 2020

Published online: 07 December 2020

\section{References}

1. Benjamin, E. J. et al. Heart disease and stroke statistics-2018 update: a report from the American heart association. Circulation 137, e67-e492. https://doi.org/10.1161/cir.0000000000000558 (2018).

2. Guh, D. P. et al. The incidence of co-morbidities related to obesity and overweight: a systematic review and meta-analysis. $B M C$ Public Health 9, 88. https://doi.org/10.1186/1471-2458-9-88 (2009).

3. National Center for Health, S. in Health, United States, 2016: With Chartbook on Long-term Trends in Health (National Center for Health Statistics (US) (2017).

4. Wildman, R. P. et al. The obese without cardiometabolic risk factor clustering and the normal weight with cardiometabolic risk factor clustering: prevalence and correlates of 2 phenotypes among the US population (NHANES 1999-2004). Arch. Intern. Med. 168, 1617-1624. https://doi.org/10.1001/archinte.168.15.1617 (2008).

5. Cornier, M. A. et al. The metabolic syndrome. Endocr. Rev. 29, 777-822. https://doi.org/10.1210/er.2008-0024 (2008).

6. Ding, C., Chan, Z. \& Magkos, F. Lean, but not healthy: the "metabolically obese, normal-weight" phenotype. Curr. Opin. Clin. Nutr. Metab. Care 19, 408-417. https://doi.org/10.1097/mco.0000000000000317 (2016).

7. Malik, S. et al. Impact of the metabolic syndrome on mortality from coronary heart disease, cardiovascular disease, and all causes in United States adults. Circulation 110, 1245-1250. https://doi.org/10.1161/01.Cir.0000140677.20606.0e (2004).

8. Samaranayake, N. R., Ong, K. L., Leung, R. Y. \& Cheung, B. M. Management of obesity in the National Health and Nutrition Examination Survey (NHANES), 2007-2008. Ann. Epidemiol. 22, 349-353. https://doi.org/10.1016/j.annepidem.2012.01.001 (2012).

9. Sherling, D. H., Perumareddi, P. \& Hennekens, C. H. Metabolic syndrome. J. Cardiovasc. Pharmacol. Ther. 22, 365-367. https:// doi.org/10.1177/1074248416686187 (2017).

10. Kip, K. E. et al. Clinical importance of obesity versus the metabolic syndrome in cardiovascular risk in women: a report from the Women's Ischemia Syndrome Evaluation (WISE) study. Circulation 109, 706-713. https://doi.org/10.1161/01.Cir.0000115514 .44135.A8 (2004).

11. Ahmadi, A. et al. Is metabolic syndrome predictive of prevalence, extent, and risk of coronary artery disease beyond its components? Results from the multinational coronary CT angiography evaluation for clinical outcome: an international multicenter registry (CONFIRM). PLoS ONE 10, e0118998. https://doi.org/10.1371/journal.pone.0118998 (2015).

12. Cavallari, I. et al. Metabolic syndrome and the risk of adverse cardiovascular events after an acute coronary syndrome. Eur. J. Prev. Cardiol. 25, 830-838. https://doi.org/10.1177/2047487318763897 (2018).

13. Reddy, V. S. et al. Relationship between serum low-density lipoprotein cholesterol and in-hospital mortality following acute myocardial infarction (The Lipid Paradox). Am. J. Cardiol. 115, 557-562. https://doi.org/10.1016/j.amjcard.2014.12.006 (2015).

14. Horwich, T. B., Hamilton, M. A., MacLellan, W. R. \& Fonarow, G. C. Low serum total cholesterol is associated with marked increase in mortality in advanced heart failure. J. Cardiac. Fail. 8, 216-224. https://doi.org/10.1054/jcaf.2002.0804216 (2002).

15. Rauchhaus, M. et al. The relationship between cholesterol and survival in patients with chronic heart failure. J. Am. Coll. Cardiol. 42, 1933-1940. https://doi.org/10.1016/j.jacc.2003.07.016 (2003).

16. Shalaurova, I., Connelly, M. A., Garvey, W. T. \& Otvos, J. D. Lipoprotein insulin resistance index: a lipoprotein particle-derived measure of insulin resistance. Metab. Syndr. Relat. Disord. 12, 422-429. https://doi.org/10.1089/met.2014.0050 (2014).

17. McGarrah, R. W. et al. A novel protein glycan-derived inflammation biomarker independently predicts cardiovascular disease and modifies the association of HDL subclasses with mortality. Clin. Chem. 63, 288-296. https://doi.org/10.1373/clinchem.2016.26163 6 (2017).

18. McGarrah, R. W. et al. High-density lipoprotein subclass measurements improve mortality risk prediction, discrimination and reclassification in a cardiac catheterization cohort. Atherosclerosis 246, 229-235. https://doi.org/10.1016/j.atherosclerosis .2016.01.012 (2016).

19. Shah, A. A. et al. Metabolic profiles predict adverse events after coronary artery bypass grafting. J. Thorac. Cardiovasc. Surg. 143, 873-878. https://doi.org/10.1016/j.jtcvs.2011.09.070 (2012)

20. Shah, S. H. et al. Baseline metabolomic profiles predict cardiovascular events in patients at risk for coronary artery disease. Am. Heart J. 163, 844-850.e841. https://doi.org/10.1016/j.ahj.2012.02.005 (2012).

21. Batch, B. C. et al. Branched chain amino acids are novel biomarkers for discrimination of metabolic wellness. Metabolism 62, 961-969. https://doi.org/10.1016/j.metabol.2013.01.007 (2013).

22. Bhattacharya, S. et al. Validation of the association between a branched chain amino acid metabolite profile and extremes of coronary artery disease in patients referred for cardiac catheterization. Atherosclerosis 232, 191-196. https://doi.org/10.1016/j. atherosclerosis.2013.10.036 (2014).

23. Shah, S. H. et al. Reclassification of cardiovascular risk using integrated clinical and molecular biosignatures: design of and rationale for the measurement to understand the reclassification of disease of Cabarrus and Kannapolis (MURDOCK) horizon 1 cardiovascular disease study. Am. Heart J. 160, 371-379.e372 (2010).

24. Executive Summary of The Third Report of The National Cholesterol Education Program (NCEP) Expert Panel on Detection, Evaluation, And Treatment of High Blood Cholesterol In Adults (Adult Treatment Panel III). Jama 285, 2486-2497 (2001).

25. Matthews, D. R. et al. Homeostasis model assessment: insulin resistance and beta-cell function from fasting plasma glucose and insulin concentrations in man. Diabetologia 28, 412-419 (1985).

26. Otvos, J. D. Measurement of lipoprotein subclass profiles by nuclear magnetic resonance spectroscopy. Clin. Lab. 48, 171-180 (2002).

27. Shah, S. H. et al. Association of a peripheral blood metabolic profile with coronary artery disease and risk of subsequent cardiovascular events. Circul. Cardiovasc. Genet. 3, 207-214. https://doi.org/10.1161/circgenetics.109.852814 (2010).

28. Kraus, W. E. et al. Metabolomic quantitative trait loci (mQTL) mapping implicates the ubiquitin proteasome system in cardiovascular disease pathogenesis. PLoS Genet 11, e1005553. https://doi.org/10.1371/journal.pgen.1005553 (2015). 
29. Duprez, D. A. et al. Comparison of the predictive value of GlycA and other biomarkers of inflammation for total death, incident cardiovascular events, noncardiovascular and noncancer inflammatory-related events, and total cancer events. Clin. Chem. 62, 1020-1031. https://doi.org/10.1373/clinchem.2016.255828 (2016)

30. Lawler, P. R. et al. Circulating N-linked glycoprotein acetyls and longitudinal mortality risk. Circ. Res. 118, 1106-1115. https:// doi.org/10.1161/circresaha.115.308078 (2016).

31. Wang, H. et al. Sildenafil treatment in heart failure with preserved ejection fraction: targeted metabolomic profiling in the RELAX trial. JAMA Cardiol. 2, 896-901. https://doi.org/10.1001/jamacardio.2017.1239 (2017).

32. Rader, D. J. \& Hovingh, G. K. HDL and cardiovascular disease. Lancet (London, England) 384, 618-625. https://doi.org/10.1016/ s0140-6736(14)61217-4 (2014).

33. Otvos, J. D. et al. Low-density lipoprotein and high-density lipoprotein particle subclasses predict coronary events and are favorably changed by gemfibrozil therapy in the Veterans Affairs High-Density Lipoprotein Intervention Trial. Circulation 113, 1556-1563. https://doi.org/10.1161/circulationaha.105.565135 (2006).

34. Lu, Y. W. et al. Lipid paradox in patients with acute myocardial infarction: potential impact of malnutrition. Clin. Nutr. (Edinburgh, Scotland) https://doi.org/10.1016/j.clnu.2018.10.008 (2018).

35. McGarrah, R. W., Crown, S. B., Zhang, G. F., Shah, S. H. \& Newgard, C. B. Cardiovascular metabolomics. Circ. Res. 122, $1238-1258$. https://doi.org/10.1161/CIRCRESAHA.117.311002 (2018).

\section{Acknowledgements}

We are grateful to all participants in the CATHGEN study.

\section{Author contributions}

S.E. conceived the presented idea, was involved with developing the data analysis plan and interpreting the data, and was primarily responsible for writing the manuscript. J.W.A. contributed to manuscript revision, data interpretation and wrote part of the manuscript. L.C.K. wrote part of the initial draft, edited the manuscript, and curated and analyzed the data. N.A.B. contributed to interpretation of data, N.P. critically revised and edited the manuscript. R.M. was involved in data interpretation, writing and editing. J.R.B. helped to plan and execute the study's metabolomics assays and critically reviewed the manuscript. W.E.K. conceived of parent project, recruited funding, obtained samples and data, review of analyses, editing of draft manuscript. S.H.S. secured funding, contributed to study design, supervised the project, and critically revised and edited the final manuscript.

\section{Funding}

Funding for this study was supported by the National Institutes of Health Grant R01-HL127009, the American Heart Association pediatric Strategically Focused Research Network Grant 17SFRN33670990, and National Heart, Lung and Blood Institute Grant 5P01-HL036587.

\section{Competing interests}

Dr. Pagidipati's work has been funded by: Amarin Pharmaceutical Company; Amgen, Inc.; AstraZeneca; Baseline Study LLC; Boehringer Ingleheim; Duke Clinical Research Institute; Eli Lilly \& Company; Novo Nordisk Pharmaceutical Company; Regeneron Pharmaceuticals, Inc.; Sanofi-S.A.; Verily Sciences Research Company. She reports consulting fees from AstraZeneca; Boehringer Ingleheim; Esperion Therapeutics. Dr. Ellison, Dr. Kwee, Dr. Bihlmeyer, Dr. Abdulrahim, Dr. McGarrah, and Dr. Bain declare no potential competing interests. Dr. Shah and Dr. Kraus have an unlicensed patent on a related research finding and Dr. Shah receives sponsored research funding from Baseline Study LLC.

\section{Additional information}

Supplementary information is available for this paper at https://doi.org/10.1038/s41598-020-78478-w.

Correspondence and requests for materials should be addressed to S.H.S.

Reprints and permissions information is available at www.nature.com/reprints.

Publisher's note Springer Nature remains neutral with regard to jurisdictional claims in published maps and institutional affiliations.

Open Access This article is licensed under a Creative Commons Attribution 4.0 International License, which permits use, sharing, adaptation, distribution and reproduction in any medium or format, as long as you give appropriate credit to the original author(s) and the source, provide a link to the Creative Commons licence, and indicate if changes were made. The images or other third party material in this article are included in the article's Creative Commons licence, unless indicated otherwise in a credit line to the material. If material is not included in the article's Creative Commons licence and your intended use is not permitted by statutory regulation or exceeds the permitted use, you will need to obtain permission directly from the copyright holder. To view a copy of this licence, visit http://creativecommons.org/licenses/by/4.0/.

(C) The Author(s) 2020 